Review Article

\title{
The effect of whole body vibration therapy on the physical function of people with type II diabetes mellitus: a systematic review
}

\author{
JiaQi Zhang, BSc ${ }^{1-3)}$, Hongyue Zhang, BSc ${ }^{1,2)}$, Laidi Kan, BSc ${ }^{1,2)}$, Chi Zhang, MD ${ }^{1,2)}$, \\ PU WANG, $\mathrm{PhD}^{1,2)^{*}}$ \\ 1) Rehabilitation Medicine Center, West China Hospital, Sichuan University: Chengdu, Sichuan \\ 610041, China \\ 2) School of Rehabilitation Sciences, West China School of Medicine, Sichuan University, China \\ 3) Faculty of Medicine, The Chinese University of Hong Kong, Hong Kong
}

\begin{abstract}
Purpose] To review and assess the effectiveness of whole body vibration therapy on the physical function of patients with type II diabetes mellitus. [Subjects and Methods] A computerized database search was performed through PubMed, Medline, EMBASE, the Cochrane Central Register of Controlled Trials, the Physiotherapy Evidence Database, and the reference lists of all relevant articles. The methodological quality was evaluated using the Physiotherapy Evidence Database scale. [Results] Five articles (four studies) with a combined study population of 154 patients with type II diabetes qualified for the inclusion criteria. Our review shows that whole body vibration therapy may have a positive impact on the muscle strength and balance of people with type 2 diabetes mellitus, whereas the effect on their mobility is still under discussion. [Conclusion] There was no sufficient evidence to support the premise that whole body vibration therapy is beneficial for the physical function of people with type II diabetes. Larger and higher-quality trials are needed.

Key words: Type II diabetes, Vibration, Systematic review
\end{abstract}

(This article was submitted Apr. 8, 2016, and was accepted May 31, 2016)

\section{INTRODUCTION}

Type 2 diabetes mellitus (T2DM) is one of the most common metabolic diseases worldwide. It is characterized by the impaired secretion or action of insulin and subsequent hyperglycemia ${ }^{1}$. People with T2DM often complain of fatigue and exhibit limitations of bodily functions ${ }^{2}$. This can profoundly affect a person's engagement in activities of daily living and reduce quality of life. T2DM can be treated with medications, with or without insulin, but it requires a multi-disciplinary approach. An active lifestyle, proper weight management, good nutrition, and avoidance of tobacco use can prevent or delay the onset and development of $\mathrm{T}_{2} \mathrm{DM}^{3}$.

Appropriate physical exercise, in addition to diet modification and medications, has been recommended as one of the three main components of diabetic management ${ }^{4}$. There is some evidence supporting the benefits of exercise performed by individuals with T2DM, such as aerobic exercise and resistance training ${ }^{5,6)}$. However, patients with T2DM are often extremely frail and unmotivated, which leads to poor compliance with physical training.

Whole-body vibration (WBV), a new physical therapeutic modality, was initially developed for use in the training of elite athletes. Now, there are numerous studies about the influence of WBV on different population subsets, such as patients with cerebrovascular accidents ${ }^{7,8}$, chronic obstructive pulmonary diseases ${ }^{9)}$, and osteoarthritis ${ }^{10)}$. These studies have revealed that WBV may have positive effects on physical functions, like mobility and balance. The effect of WBV is thought to be

\footnotetext{
*Corresponding author. Pu Wang (E-mail: wangpu_03@126.com)

(C)2016 The Society of Physical Therapy Science. Published by IPEC Inc.

This is an open-access article distributed under the terms of the Creative Commons Attribution Non-Commercial No Derivatives (by-nc-nd) License $<$ http://creativecommons.org/licenses/by-nc-nd/4.0/>.
} 
mediated by muscle contraction, facilitation of sensory inputs, and stimulation of proprioceptive receptors ${ }^{11)}$. In recent years, an increasing number of studies have examined whether or not WBV is truly beneficial for people with $\mathrm{T}_{2} \mathrm{DM}^{12-19)}$, but the evidence still shows inconsistencies. The aim of this review was to systematically assess randomized controlled trials featuring the application of WBV aiming to improve the physical function of people with T2DM.

\section{SUBJECTS AND METHODS}

A literature search for relevant studies was conducted on MEDLINE (1966 to Jul 2015; via Ovid), the Cochrane Central Register of Controlled Trials (CENTRAL) (The Cochrane Library, Issue 7 of 12 Jul 2015), PubMed (1966 to Jul 2015), Physiotherapy Evidence Database (PEDro) (1929 to Jul 2015; via website), and EMBASE (1980 to Jul 2015; via Ovid). The keywords used for searching were diabetes mellitus or diabetes or DM and vibration or whole body vibration or WBV or biomechanical stimulation and randomized controlled trial or clinical trial or controlled clinical trial or trial or randomized or randomly or placebo. The reference lists of each selected article were manually searched to identify other potentially relevant papers. The latest search was performed on 18th December, 2015, on Pubmed to update the study. Studies that met the requirements using the following criteria were considered for review: (1) randomized controlled trials (RCTs) concerning the effect of WBV, with or without physical activity, on people diagnosed with T2DM; (2) publication in English; (3) WBV aiming improve the physical function (e.g. mobility) of people with T2DM; and (4) the intervention in the control arm of the study included a sham WBV intervention, exercise, or other conventional treatment modalities. Articles were excluded if they were: (1) studies conducted on patients with other primary diagnoses (e.g. stroke); (2) reports published as conference proceedings; or (3) published in books. Two authors independently assessed each study's risk of bias according to the Physiotherapy Evidence-Based Database (PEDro) scale ${ }^{20)}$. The PEDro scale consists of 10 quality ratings, each receiving either a yes or no. The ten items examined were random allocation, concealment of allocation, baseline equivalence, blind therapists, blind subjects, blind assessors, intention to treat analysis, adequacy of follow-up, between-group statistical analysis, point estimates variability. When disagreement existed between two reviewers, scoring discrepancies were resolved through discussion.

\section{RESULTS}

The initial database searches retrieved a total of 244 articles, of which 40 records were excluded due to duplication. After reading the titles and abstracts, 15 full articles were retrieved. Ten were subsequently eliminated for the following reasons. three of them did not have any outcome measures related to physical function, two were pre-post studies, three were case studies, one was a conference abstract without full text, and the other was not related to our topic. Only five articles (4 studies) that met all the eligibility criteria were included in this review (Table 1, Fig. 1). The assessment of the methodological quality is provided in Table 2. Only one study ${ }^{16}$ ) was considered to be a good quality trial (PEDro 6-7), and the rest were considered to be fair (PEDro 4-5).

Four articles (three studies) ${ }^{16-19)}$ assessed balance and mobility using to the timed up and go test (TUG). Their results showed that the WBV groups demonstrated a significant treatment effect when compared to the control groups. This was regardless of the treatment duration, parameters of $\mathrm{WBV}$, and characteristics of the participants (WMD, $-0.79 ; 95 \% \mathrm{CI}$, -1.16 to $-0.43 ; \mathrm{p}<0.0001, \mathrm{p}$ for heterogeneity $<0.00001)$, with strong evidence for statistical heterogeneity $\left(\mathrm{I}^{2}=93 \%\right)(\mathrm{Fig}$. 2). Subgroup analysis was done for further analysis Meta-analysis of the TUG results revealed a significant improvement in favor of six-week WBV for patients with diabetic neuropathy. (WMD $=-1.41,95 \% \mathrm{CI}=-1.84$ to $-0.98, \mathrm{p}<0.00001, \mathrm{p}$ for heterogeneity $=0.29, \mathrm{I}^{2}=12 \%$ ).

\section{DISCUSSION}

This review assessed the effect of WBV on the functional performance of people with T2DM. Although a majority of these studies reported a favorable effect following WBV therapy, some inconsistencies were found in this review.

Five articles (four studies) explored the different aspects of the application of WBV therapy for people with T2DM. Baum $\mathrm{K}$ et al. ${ }^{15)}$ compared whole body vibration with exercise (WBVE) therapy with other kinds of exercise training (WBV plus exercise vs. exercise); del Pozo-Cruz J et al. and del Pozo-Cruz B et al. ${ }^{17}{ }^{18)}$ focused on the effect of WBVE (WBV plus exercise vs no physical training); and Lee $\mathrm{K}$ et al. ${ }^{16)}$ analyzed both of these. The main outcome measures related to physical function were muscle strength, balance, and mobility.

Three studies assessed the muscle strength using different outcome measures ${ }^{16,18,19)}$. Using the five times sit-to-stand (FTSTS) test, Lee $\mathrm{K}$ et al. ${ }^{16)}$ reported a positive influence on the lower limb strength of patients with diabetic neuropathy who underwent six-weeks of WBV with a balanced exercise program compared to a control group. del Pozo-cruz B et al. ${ }^{18)}$ also reported a significant improvement in the 30s sit-to-stand (30s-STS) test in favor of WBV. Kordi Yoosefinejad A et al. ${ }^{19)}$ reported that the isometric strengths of both the tibialis anterior and quadriceps, which were measured using a back-leg-chest dynamometer, improved after a six-week WBV intervention compared to a control group. Muscle strength should be the primary outcome assessed, and muscle strength improvement is the most likely outcome to be influenced by 


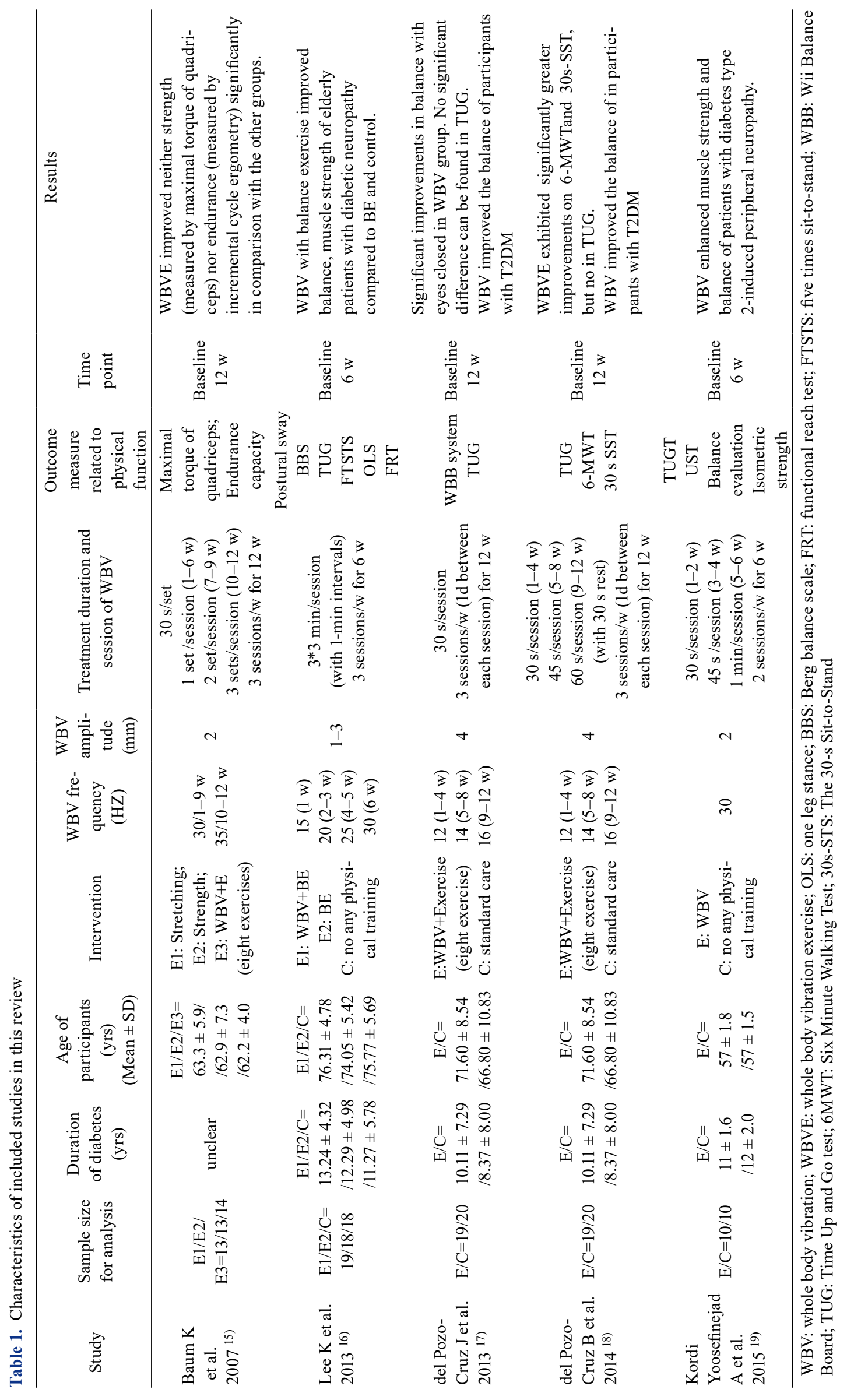




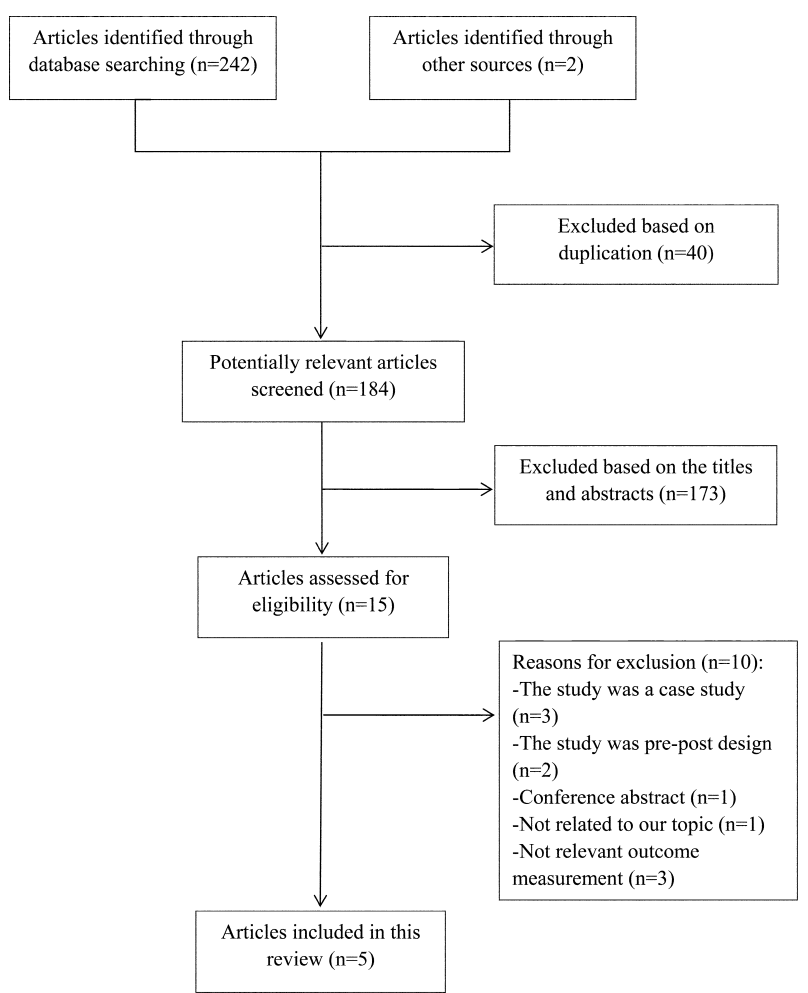

Fig. 1. Search strategy and flow chart for this meta-analysis RCT: randomized controlled trial

Table 2. Assessment of the methodology using the PEDro Scale*

\begin{tabular}{|c|c|c|c|c|c|}
\hline Criterion & $\begin{array}{c}\text { Baum K et al. } \\
2007^{15)}\end{array}$ & $\begin{array}{c}\text { Lee K } \\
\text { et al. 2013 }\end{array}$ & $\begin{array}{l}\text { del Pozo-Cruz J } \\
\text { et al. 2013 }\end{array}$ & $\begin{array}{l}\text { del Pozo-Cruz B } \\
\text { et al. 2014 }\end{array}$ & $\begin{array}{c}\text { Kordi Yoosefinejad } \\
\text { A et al. 2015 }\end{array}$ \\
\hline Eligibility criteria & No & Yes & Yes & Yes & Yes \\
\hline Random allocation & 1 & 1 & 1 & 1 & 1 \\
\hline Concealed allocation & 0 & 0 & 0 & 0 & 1 \\
\hline Baseline comparability & 1 & 1 & 1 & 1 & 1 \\
\hline Blind subjects & 0 & 0 & 0 & 0 & 0 \\
\hline Blind therapists & 0 & 0 & 0 & 0 & 0 \\
\hline Blind assessors & 0 & 1 & 0 & 1 & 0 \\
\hline Adequate follow-up & 0 & 1 & 0 & 0 & 0 \\
\hline Intention-to-treat analysis & 0 & 0 & 0 & 0 & 0 \\
\hline Between group comparisons & 1 & 1 & 1 & 1 & 1 \\
\hline Point estimates and variability & 1 & 1 & 1 & 1 & 1 \\
\hline Total scores & 4 & 6 & 4 & 5 & 5 \\
\hline
\end{tabular}

*The PEDro scores were taken from the PEDro website

vibration-induced muscular reflex ${ }^{11)}$. All the results indicate that WBV with or without exercise training has positive effects on the lower extremity muscle strength of people with T2DM.

The improvement of muscle strength may contribute to the enhancement of balance and mobility, but some inconsistencies were still found in this respect.

Various balance assessments were mentioned in the included studies, including a Wii balance board ${ }^{17)}$, the Berg balance scale (BBS), the functional reach test (FRT) ${ }^{16}$, and the one leg stance (OLS)/unilateral stance test (UST) ${ }^{16,19)}$. Most studies reported a positive results in the different balance assessments in favor of WBV, except the study by Kordi Yoosefinejad A et al. ${ }^{19)}$, which reported that there was no significant difference between the WBV group and the control group according to the 


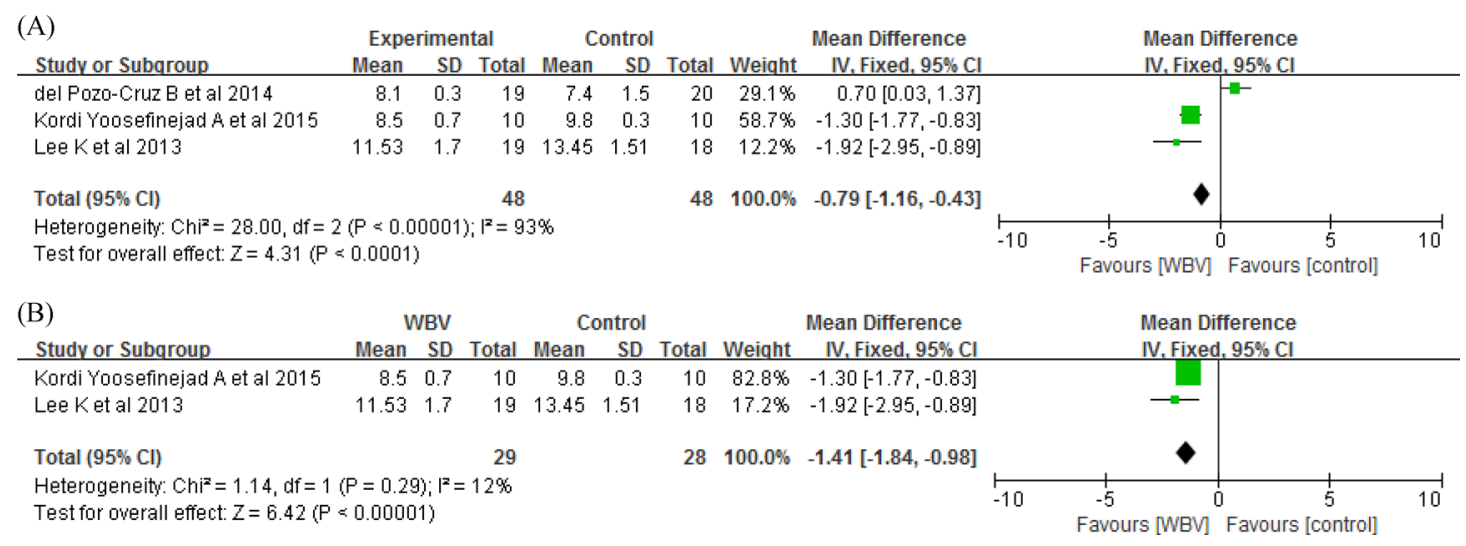

Fig. 2. (A) The aggregated results of TUG of three studies. (B) The aggregated results of TUG of two studies: Six-week intervention

UST. del Pozo-Cruz J et al. ${ }^{17)}$ reported that WBV therapy improved balance performance when the participants' eyes closed, which meant that WBV may have stimulated proprioceptive responses, and helped the subjects to acquire a better balance performance ${ }^{21,22)}$. The TUG can assess a person's mobility, and it requires both static and dynamic balance ${ }^{23)}$. Lee K et al. ${ }^{16)}$ and Koedi Yoosefinejad A et al. ${ }^{19)}$ reported a significant improvement in the TUG time after WBV therapy, but del Pozo-Cruz $\mathrm{B}$ et al. ${ }^{18)}$ reported an inconsistent result. The meta-analysis of the present study found that the pooled result showed a significant improvement in favor of a six-week WBV for patients with diabetic neuropathy. A possible explanation for this may be that the participants in the studies by Lee et al. ${ }^{16)}$ and Koedi Yoosefinejad A et al. ${ }^{19)}$ all suffered peripheral neuropathy, which may be the major reason behind the deterioration in mobility and dynamic balance, and hence, the changes were more obvious. In addition, the optimal outcome measure of choice for people with T2DM, with or without neuropathy, is not well studied, and the sensitivity and reliability of the TUG test of people with T2DM without neuropathy may have some bias.

Besides the problem with balance that people with T2DM have, issures concerning falls caused by limited balance deserve more attention. In the studies reviewed, only Lee et al. ${ }^{16)}$ discussed the possible effects of vibration on the risk of fall, which is strongly related to community mobility and social participation. Instead of achieving normal physical function, the activity and participation of people with T2DM deserve more attention ${ }^{24)}$. An example worthy of further research would be instrumental activities of daily living. Patients with T2DM frequently suffer progressive functional degeneration in many aspects $^{25)}$, and taking this fact into consideration, there is still a lack of evidence to support the long-term effects of WBV therapy on the functional capacity of patients with T2DM. Adequately prolonged follow-up studies are required.

In addition, numerous studies did not follow the recommendations of the International Society of Musculoskeletal and Neuronal Interactions ${ }^{26}$. This may have resulted in bias due to that missing information relating to the intervention parameters and treatment plans. It is, therefore, strongly recommended that future authors reporting on WBV therapy comply with these recommendations.

There were three limitations of this systematic review and meta-analysis. First, our meta-analysis is used a only small number of RCTs focused on this area, therefore the conclusions should be interpreted with caution. Second, the protocol of WBV and the participants varied greatly, so more quantitative analyses could not be performed. Third, exclusion of nonEnglish language studies and some missing and unpublished data may have resulted in bias.

As a novel, effective, safe, and alternative approach, WBV therapy may be made available in rehabilitation programs for the management of T2DM. However, variability in the parameters of WBV therapy can be found, including differences in the levels of vibration and the duration of the interventions. Moreover, the characteristics of each patient should also be taken into consideration, such as whether they have diabetic neuropathy or not. Overall, the evidence for the application of WBV therapy for the physical function of patients with T2DM is still inconclusive. More trials using a more specific therapy regimen for WBV therapy for people with T2DM are needed.

\section{ACKNOWLEDGEMENTS}

This manuscript is the original work of the authors and has not been submitted for publication before. Part of the material in this manuscript was accepted for poster presentation at the 5th Asia-Oceanian Conference of Physical and Rehabilitation Medicine and the 26th Philippine Academy of Rehabilitation Medicine Annual Convention, Cebu, Philippines. This work is supported by National Natural Science Foundation of China (81401858). 


\section{REFERENCES}

1) Kahn SE, Cooper ME, Del Prato S: Pathophysiology and treatment of type 2 diabetes: perspectives on the past, present, and future. Lancet, 2014, 383: 10681083. [Medline] [CrossRef]

2) Gregg EW: Diabetes-related disability as a target for prevention. Lancet Diabetes Endocrinol, 2013, 1: 81-82. [Medline] [CrossRef]

3) Global status report on noncommunicable diseases 2010: Geneva: World Health Organization, 2011.

4) Sanz C, Gautier JF, Hanaire H: Physical exercise for the prevention and treatment of type 2 diabetes. Diabetes Metab, 2010, 36: 346-351. [Medline] [CrossRef]

5) Jorge ML, de Oliveira VN, Resende NM, et al.: The effects of aerobic, resistance, and combined exercise on metabolic control, inflammatory markers, adipocytokines, and muscle insulin signaling in patients with type 2 diabetes mellitus. Metabolism, 2011, 60: 1244-1252. [Medline] [CrossRef]

6) Abd El-Kader SM: Aerobic versus resistance exercise training in modulation of insulin resistance, adipocytokines and inflammatory cytokine levels in obese type 2 diabetic patients. J Adv Res, 2011, 2: 179-183. [CrossRef]

7) Yang X, Wang P, Liu C, et al.: The effect of whole body vibration on balance, gait performance and mobility in people with stroke: a systematic review and meta-analysis. Clin Rehabil, 2015, 29: 627-638. [Medline] [CrossRef]

8) Lu J, Xu G, Wang Y: Effects of whole body vibration training on people with chronic stroke: a systematic review and meta-analysis. Top Stroke Rehabil, 2015, 22: 161-168. [Medline] [CrossRef]

9) Yang X, Zhou Y, Wang P, et al.: Effects of whole body vibration on pulmonary function, functional exercise capacity and quality of life in people with chronic obstructive pulmonary disease: a systematic review. Clin Rehabil, 2016, 30: 419-431. [Medline] [CrossRef]

10) Zafar H, Alghadir A, Anwer S, et al.: Therapeutic effects of whole-body vibration training in knee osteoarthritis: a systematic review and meta-analysis. Arch Phys Med Rehabil, 2015, 96: 1525-1532. [Medline] [CrossRef]

11) Cardinale M, Bosco C: The use of vibration as an exercise intervention. Exerc Sport Sci Rev, 2003, 31: 3-7. [Medline] [CrossRef]

12) Mohammad-Ali A, Behboudi L, Aghaalinejad H, et al.: Comparative effects of aerobic training and whole body vibration on plasma adiponectin and insulin resistance in type 2diabetic men. Ann Biol Res, 2011, 2: 671-680.

13) Behboudi L, Azarbayjani MA, Aghaalinejad H, et al.: Effects of aerobic exercise and whole body vibration on glycaemia control in type 2 diabetic males. Asian J Sports Med, 2011, 2: 83-90. [Medline] [CrossRef]

14) Sañudo B, Alfonso-Rosa R, Del Pozo-Cruz B, et al.: Whole body vibration training improves leg blood flow and adiposity in patients with type 2 diabetes mellitus. Eur J Appl Physiol, 2013, 113: 2245-2252. [Medline] [CrossRef]

15) Baum K, Votteler T, Schiab J: Efficiency of vibration exercise for glycemic control in type 2 diabetes patients. Int J Med Sci, 2007, 4: 159-163. [Medline] [CrossRef]

16) Lee K, Lee S, Song C: Whole-body vibration training improves balance, muscle strength and glycosylated hemoglobin in elderly patients with diabetic neuropathy. Tohoku J Exp Med, 2013, 231: 305-314. [Medline] [CrossRef]

17) Del Pozo-Cruz J, Alfonso-Rosa RM, Ugia JL, et al.: A primary care-based randomized controlled trial of 12-week whole-body vibration for balance improvement in type 2 diabetes mellitus. Arch Phys Med Rehabil, 2013, 94: 2112-2118. [Medline] [CrossRef]

18) del Pozo-Cruz B, Alfonso-Rosa RM, del Pozo-Cruz J, et al.: Effects of a 12-wk whole-body vibration based intervention to improve type 2 diabetes. Maturitas, 2014, 77: 52-58. [Medline] [CrossRef]

19) Kordi Yoosefinejad A, Shadmehr A, Olyaei G, et al.: Short-term effects of the whole-body vibration on the balance and muscle strength of type 2 diabetic patients with peripheral neuropathy: a quasi-randomized-controlled trial study. J Diabetes Metab Disord, 2015, 14: 45. [Medline] [CrossRef]

20) Physiotherapy Evidence Database (PEDro): (1999): PEDro scale. Retrieved November 3, 2010. http: // www.pedro.org.au/english/downloads/pedro-scale/.

21) Osawa Y, Oguma Y, Ishii N: The effects of whole-body vibration on muscle strength and power: a meta-analysis. J Musculoskelet Neuronal Interact, 2013, 13: 380-390. [Medline]

22) Lau RW, Liao LR, Yu F, et al.: The effects of whole body vibration therapy on bone mineral density and leg muscle strength in older adults: a systematic review and meta-analysis. Clin Rehabil, 2011, 25: 975-988. [Medline] [CrossRef]

23) Podsiadlo D, Richardson S: The timed "Up \& Go": a test of basic functional mobility for frail elderly persons. J Am Geriatr Soc, 1991, 39: 142-148. [Medline] [CrossRef]

24) Poole JL, Gashytewa C, Sullivan AT: Activity limitations, participation, and quality of life in American Indians with and without diabetes. Occup Ther Health Care, 2016, 30: 58-68. [Medline] [CrossRef]

25) Botelhoa MC, Conde MG, Rebelo Braz NM: Functional aspects in ageing adults with diabetic neuropathy. A review. Curr Diabetes Rev, 2015. [CrossRef]

26) Rauch F, Sievanen H, Boonen S, et al. International Society of Musculoskeletal and Neuronal Interactions: Reporting whole-body vibration intervention studies: recommendations of the International Society of Musculoskeletal and Neuronal Interactions. J Musculoskelet Neuronal Interact, 2010, 10: 193-198. [Medline] 